\title{
A Simple, Inexpensive 1-D Haptic Tool
}

\author{
Anupama Thomas, Prabhav N. Reddy, and George Mathew
}

\begin{abstract}
Haptics refers to recognizing objects through tactile feedback and haptic technology refers to applying forces to the user's hand, allowing the user to "feel" objects in a virtual environment. Haptics technology is an emerging field in developing countries and is being increasingly used in simulation and virtual training environment. Since haptic applications use specialized hardware to provide tactile feedback to the user, the costs are considerable limiting the study of haptics and the processes of designing haptic devices in general. In this paper, a simple, inexpensive single dimensional haptic device is presented. This simple design offers a basic didactic platform to explore and study haptic interfaces. The role of haptics in surgical simulators has been studied and how this simple tool can be modified to function as a basic simulator tool has been explored. In providing this simple design, users can experiment with possible variations with ease.
\end{abstract}

Index Terms-Haptics, haptic tool, simulation.

\section{INTRODUCTION}

Haptics introduces a rich bidirectional sensorial layer to various interactions. Developing hardware and software that provides a realistic haptic experience is still expensive. Over the years much work has been done in the field of haptics to enable designers to explore and discuss various aspects of haptics. Moussette and Banks [1] designed a few simple haptic devices such as the Slacker, Springer and the Winder providing a platform for an increased understanding of haptics. Hayward and Maclean [2] described the technical challenges associated with building haptic interfaces. Komerska and Ware [3] used simple haptic force constraints and a visually intuitive environment to create a hapticallyenhanced sculpting application.

Haptics plays an important part in minimally invasive surgical training in a simulated environment. Minimally invasive surgery has transformed various surgical procedures over the past few years. In this mode of surgery, long slender tools are introduced into the abdominal cavity of the patient through a small incision. Surgeons can operate on the internal organs from outside without opening the abdominal cavity as

Manuscript received May 9, 2013; revised July 24, 2013. This work was supported by the Department of Information Technology, Government of India.

Anupama Thomas is with the Department of Bioengineering, Christian Medical College,Vellore, Tamil Nadu, India (e-mail: reganupthomas@ cmcvellore.ac.in).

Prabhav N Reddy is with the School of Bio- Sciences and Technology, Vellore Institute of Technology, Vellore, TamilNadu,India (e-mail: nrprabhav@gmail.com).

George Mathew is the Executive Dean, Faculty of Medicine and Medical School, Universitas Pelita Harapan, Jl. Boulevard Jen Sudirman, Lippo Karawaci Tangerang 15811-Indonesia (e-mail: gmathewvellore@ yahoo. com). in conventional surgery while viewing the organs and the manipulations by the instruments on a video display. These types of surgeries cause less inconvenience to the patient in terms of considerably reduced post-operative pain leading to faster recovery and reduced hospital stay, yet because of a decreased sense of touch as compared to an open surgery, surgeons need longer periods of training to master this technique and successfully operate on patients. Traditionally training for minimally invasive surgery is by apprenticeship. Now progressively training for minimally invasive surgeries is done by way of simulations where virtual reality training tools are used to enhance the surgeons' skill level. There are many available surgical simulations where only a visual feedback is given to the trainee without any force feedback. Studies [4] have shown that a visual feedback accompanied with a haptic feedback would enhance the skill level of surgical trainees. The role of haptics in Minimally Invasive Surgical Skills training has been explored [5], [6] where haptic devices have been integrated into training systems which have been designed for minimally invasive procedures.

Currently, haptic devices that are used in areas such as haptic research and surgical simulation are very expensive costing over US\$10,000. These render small forces to the user through a complex system of servo engines and mechanical links. The most popular ones are Sensable's PHANToM $^{\mathrm{R}} \mathrm{Omni}^{\mathrm{TM}}$ and Desktop ${ }^{\mathrm{TM}}$ [7] that apply forces to the user in the shape of a stylus.

Falcon Novint [8] has been released in the USA in conjunction with computer games. As opposed to traditional haptic devices that use motors, linkages and bearings. ButterflyHaptics $^{\mathrm{TM}}$ [9] uses the principle of magnetic levitation in the design of its magnetic levitation haptic device known as Maglev $200{ }^{\mathrm{TM}}$ Haptic Interface.

Many visuo-haptic simulation systems are designed for complex procedures such as vertebroplasty have been simulated for medical student training in spinal cement vertebroplasty procedure [10]. The task of needle insertion involved in spinal injections has also been simulated [11].

Instead of replicating the entire procedure virtually, it has been seen that training in part -tasks, such as the MIST-VR (Minimally Invasive Surgery Trainer-Virtual Reality) can also enhance the skill of trainees[12], [13].The MIST-VR provides trainees with an assessable environment. In the MIST, simple tasks/part-tasks have been designed to simulate basic manoeuvres performed during surgical procedures. It presents an abstract concept of the surgical workspace consisting of simple geometries and allowing very simple interactions. Even though this has the lowest realism of the surgical scene yet it allows trainees to acquire basic skills needed for performing surgeries.

In this paper we present a simple design of a 1-D haptic 
tool. This can be further modified by the addition of two additional motors with similar control electronics to give a force feedback in 3 degrees of freedom and simulate simple part-task training scenarios for surgical trainees. The haptic tool (consisting of a Joystick, a potentiometer, a BLDC motor) which works together with a computer and custom-built software forms the haptic system (Fig. 1). The cost of this tool is less than $\$ 400$.

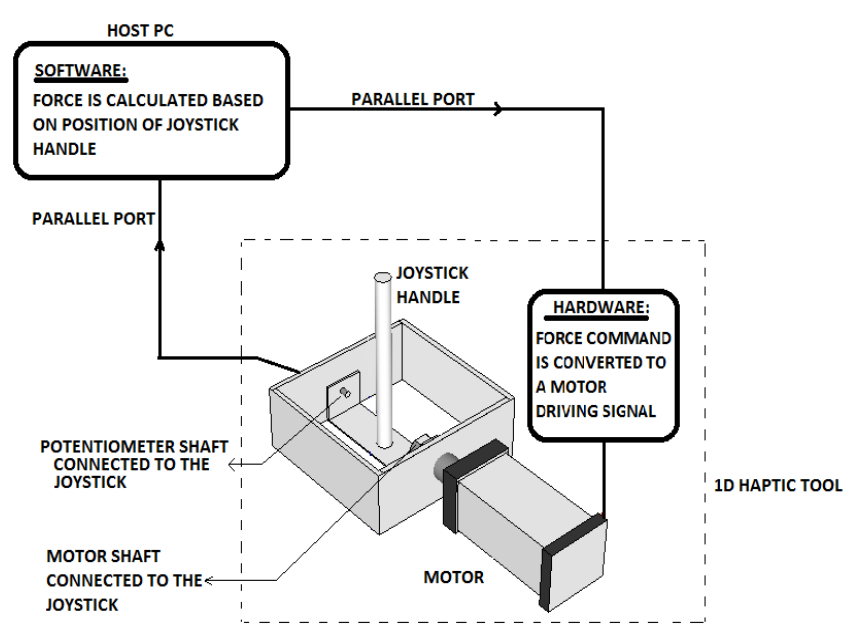

Fig. 1. The haptic system

\section{Design AND Details}

The haptic system can simulate a virtual surface as perceived by a probing finger or hand. This can be achieved using a displacement sensor to detect the finger position and then presenting an appropriate resisting force using a motor. This is the force is felt by the probing hand of the user on the joystick handle.

The sequence of operations is as follows : (a) displacement of the probing finger/hand is sensed by the potentiometer, (b) the position is read by the computer, and the changes to the graphical scene are drawn,(c) depending on the hardness of the object in the virtual environment the computer commands the motor to present the required force.

The joystick handle is connected on one side to the rotary potentiometer and on the other side to the shaft of the electronically controlled motor as shown above. The rotary potentiometer senses the displacement of the joystick while the electronically controlled motor presents the required forces. The joystick, rotary potentiometer and the motor together comprise the 1-D haptic tool.

Calculation of the appropriate forces is done by a computer. The virtual environment scene is presented graphically on the computer screen.

The host $\mathrm{PC}$ reads the value of the current position of the potentiometer through the parallel port and the force command, as a function of position of the joystick handle, is sent back to the electronics through the parallel port and converted to a motor driving signal. This is felt as a force on the joystick handle by the user. A flowchart of the software program flow is given (Fig. 2).

The Hurst BLDC motor was used to present a constant force to the user. Since a BLDC motor cannot commutate the windings, the control circuit and the software must control the current flow correctly to keep the motor turning smoothly. The dsPIC 30F3011 was used to develop code to control the BLDC motor. Hall sensors embedded in the motor sensed the current rotor position and the windings were energized in sequence according to a specific look -up table.

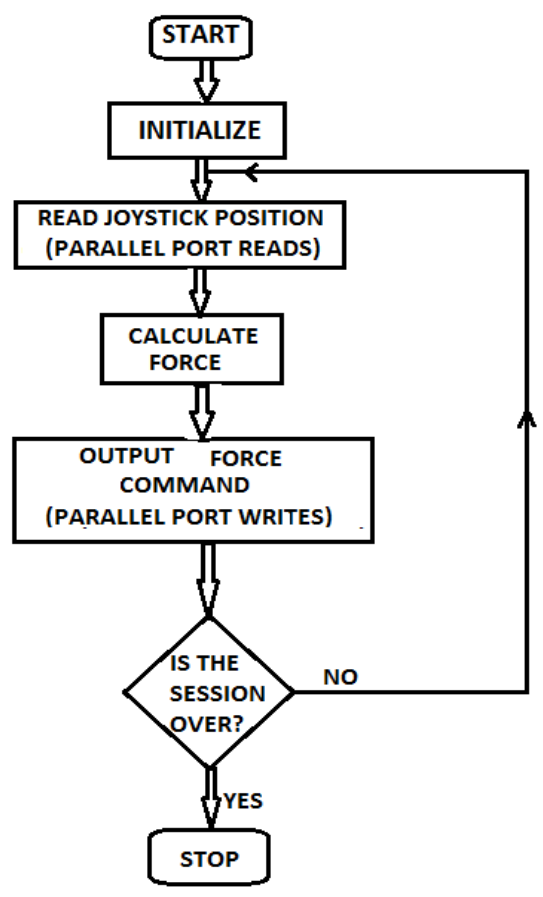

Fig. 2. Flowchart of software

To present a constant force to the user, the current flowing through the motor was monitored, since force is proportional to the current flowing through it. For a constant force application, the actual current is compared with a set reference. The error was amplified using the PID algorithm. The proportional gain was adjusted to get the best transient and steady state responses. The amplified error was then used to readjust the PWM duty cycle so that even on loading the motor, the current flowing through the motor would remain within limits (Fig. 3).

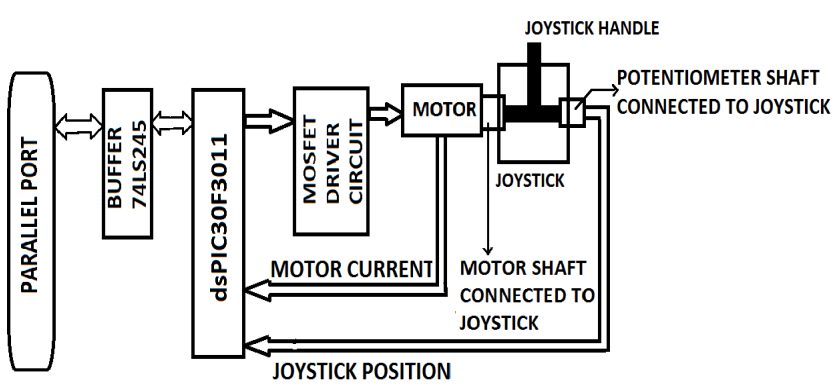

Fig. 3. Block diagram of the control electronics for the motor

The force command is sent to the motor from the virtual environment scene through the parallel port. When set in the EPP mode, the speed of data transfer is $2 \mathrm{Mbytes} / \mathrm{sec}$.The graphical scene was generated using $\mathrm{VC}++$ and OpenGL.

Forces of up to $2.2 \mathrm{~N}$ can be felt by the user using this haptic tool. To measure the forces presented by the motor, weights of different values were kept on a rod (attached to the motor shaft) at a distance of $6 \mathrm{~cm}$ from the center of the shaft. Over a range of input voltages ( 0 to $5.5 \mathrm{~V})$ given as motor- 
driving signals through the microcontroller, different weights were added to stop the shaft from moving. This provided the values of resisting forces presented by the motor at varying input voltages. These are the range of forces felt by the user during the haptic session.

\section{RESULTS AND DiSCUSSION}

The graphics in the host PC shows 2 spheres in the virtual environment (Fig. 5a, 5b). The smaller yellow sphere represents the probing hand of the user and its movement is picked up by the potentiometer shaft/joystick handle. The large red sphere is an object in the virtual environment. As the joystick handle is manipulated, the smaller sphere touches the bigger sphere. When the two spheres meet, a motor driving signal of $2 \mathrm{~V}$ is sent by the graphics software module to the micro-controller and a force of almost $1 \mathrm{~N}$ ( as shown in the force-voltage profile in Fig. 4 ) is the resisting force felt by the user, which corresponds to the hardness of the red ball felt on the joystick handle.

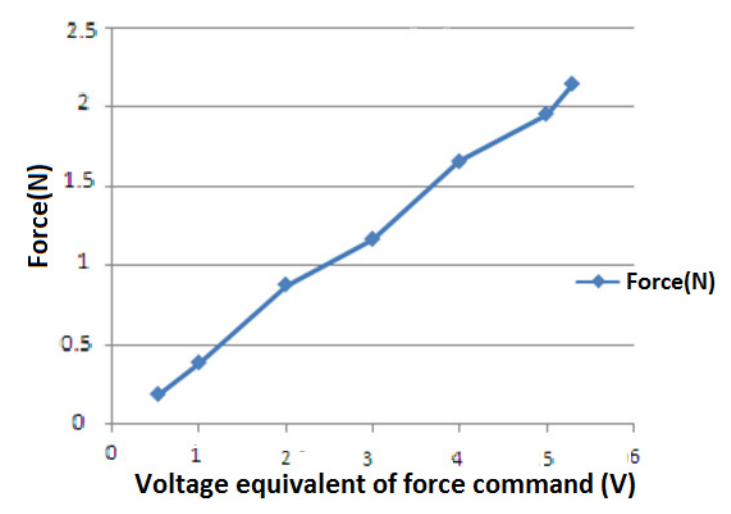

Fig. 4. Force-Voltage profile of the haptic tool

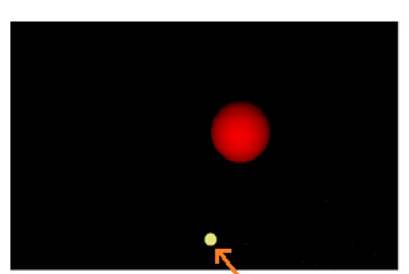

(a) YELLOW SPHERE MOVES AS JOYSTICK IS MANIPULATED

(b)

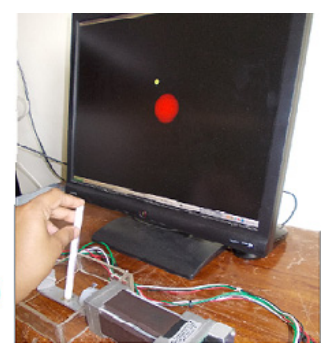

Fig. 5. (a) Graphics of the haptic system (b) Screenshot of the haptic system
Overall, using this simple haptic tool provided a valuable platform for an understanding of haptics. The ability to "feel" haptics is needed in designing haptic systems even if the simulations are very basic. This 1-D haptic tool has demonstrated force feedback in one dimension.

In future, the haptic tool can be further developed to include 2 additional motors and give a combined force feedback in 3 degrees of freedom. This will have its application in basic virtual surgical simulators where the graphics can be modified to feature part- task training modules. The software code can be modified to experiment with new haptic rendering algorithms.

\section{REFERENCES}

[1] C. Moussette and R. Banks, "Designing through making: exploring the simple haptic design space," in Proceedings of TEI'11, Tangible, Embedded and Embodied Interaction 2011, Funchal, Portugal, January 2011, pp. 22-26.

[2] V. Hayward and K. E. MacLean, "Do it yourself haptics, Part-I," IEEE Robotics and Automation Magazine, vol. 14, no.4, pp. 88-104, 2007.

[3] R. Komerska and C. Ware, "Haptic-GDraw: A fun and easy to use 3D haptically-enhanced sculpting," in Proceedings of EuroHaptics, Munich Germany, June 5-7, 2004.

[4] H. K. Kim, D. W. Rattner, and M. A. Srinivasan,"The role of simulation fidelity in laparoscopic surgical training," in Medical Image Computing and Computer-Assisted Intervention - MICCAI 2003 Lecture Notes in Computer Science, vol. 2878, pp. 1-8, 2003.

[5] F. G. Hamza-Lup, C. M. Bogdan, D. M. Popovici, and O. D. Costea, "A survey of visuo-haptic simulation in surgical training," presented at the International Conference on Mobile, Hybrid, and On-line Learning, Gosier, Guadeloupe, France, 22-28 February, 2011.

[6] Basdogan C., S. De, J. Kim, M. Manivannan, H. Kim and M.A. Srinivasan,"Haptics in minimally invasive surgical simulation and training "IEEE Computer Graphics and Applications (IEEE CG\&A), March 2004.

[7] Sensable Haptics [Online] Available: http://www.sensable.com

[8] Novint Haptics [Online] Available: http://www.novint.com

[9] Butterfly haptics [Online] Available: http://www.butterflyhaptics.com

[10] C. K Chui, J. S. K Ong, Z. Y. Lian, Z. Wang, and J. Teo, "Haptics in computer- mediated simulation: training in vertebroplasty surgery," Simulation and Gaming, vol. 37, pp. 438-451, 2006.

[11] M. Farcas, "Development of a virtual reality haptic veress needle insertion simulator for surgical skills training," presented at Medicine Meets Virtual Reality, Longbeach, California, Jan.19-22, 2009.

[12] P. Lamata, J. Enrique Gómez, F. Bello, R. L. Kneebone, R. Aggarwal, and F. Lamata, "Conceptual framework for laparoscopic VR simulators," IEEE Computer Graphics and Applications, vol. 26, no. 6, pp.69-79, 2006.

[13] N. E. Seymour, A. G. Gallagher, S. A. Roman et al, "Virtual reality training improves operating performance: results of a randomized, double - blinded study," Annals Surgery, vol. 236, no.4, pp. 458-463, 2002 .

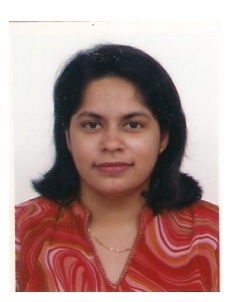

Anupama Thomas obtained her Bachelor's degree in Electrical and Electronics Engineering from the College of Engineering, Trivandrum, India in 1996. She completed her Master's degree in Biomedical Engineering from the Manipal Institute of Technology, Manipal, India in 1999.

Her current research interests include Haptics, Rehabilitation Engineering and Medical Instrumentation.

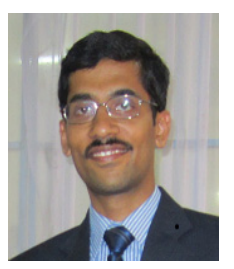

Prabhav N. Reddy obtained his Bachelor's degree in Electronics and Communications from the Indian Institute of Technology, Guwahati, India in 2004. He completed his Master's degree in Biomedical engineering from the Indian Institute of Technology, Bombay, India in 2006.

His research interests include Rehabilitation Engineering, Medical instrumentation and Signal processing.

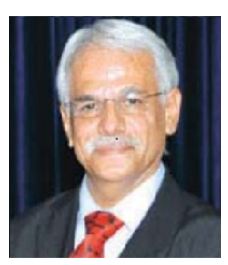

George Mathew completed his Bachelor's degree in Medicine from the Christian Medical College, Vellore, India. He went on to obtain the Master of Surgery degree in 1984 also from the Christian Medical College, Vellore. He trained at the Royal Adelaide Centre for Endoscopic surgery, Australia. He is a skilled surgeon in performing complex laparoscopic procedures. Currently, he is the President, Medical Sciences, UPH President, MIRIN, (Mochtar Riady Institute for Nanotechnology) Executive Dean Faculty of Medicine\& Medical School, Universitas Pelita Harapan, Indonesia.

Dr. George Mathew has won numerous awards for his skill and competence in performing laparoscopic surgery. 